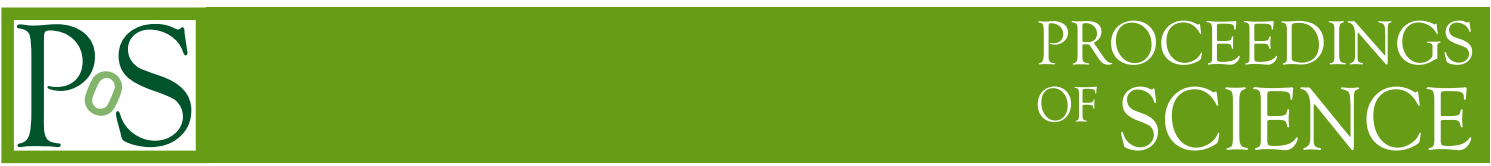

\title{
The TRISTAN Antarctic Cosmic Ray detector
}

\section{García-Castro*, on behalf of TRISTAN Collaboration ${ }^{\dagger}$}

Instituto Galego de Física de Altas Enerxías, University of Santiago de Compostela, Spain

E-mail: damian.garcia@usc.es

\begin{abstract}
TRISTAN detector is part of a new family of high accuracy multitracking capabilities. TRISTAN was designed to be installed at the Spanish Antartic Base in Livignston Island, as one of the dectectors of ORCA (Antarctic Cosmic Ray Observaroty). It will be complemented by a neutron monitor and a muon telescope with the purpose of mesuring cosmic rays at different latitudes. Preliminary results on the study of rates of cosmic rays measured during the two-way journey from Vigo (Spain) to Punta Arenas (Chile) are shown in this paper.
\end{abstract}

36th International Cosmic Ray Conference -ICRC2019-

July 24th - August 1st, 2019

Madison, WI, U.S.A.

\footnotetext{
* Speaker.

${ }^{\dagger}$ for collaboration list see PoS(ICRC2019)1177
} 


\section{Introduction}

Most of cosmic rays are relativistic charged atomic nuclei, which travel through the space and hit the Earth's atmosphere. They are originated outside the Earth and due to distortions in their trajectories, caused by magnetic fields in the interstellar space, they arrive to the Earth's atmosphere. Around 90\% are protons, 9\% are alpha particles and the rest is a contribution of medium and heavy nuclei and they are distinguished by their high energies. Most of them have ultra-relativistic energies that are up to around $10^{20} \mathrm{eV}$, eleven orders of magnitude greater than the energy of the proton in rest and whose origin is external to our galaxy.

The variations in the cosmic rays flux detected are affected by different effects, as the interplanetary magnetic field induced by the Sun, the solar wind, the Earth's magnetic field or the atmosphere.

Cosmic rays hit the Earth's atmosphere regularly, interacting with atmospheric target nuclei and the subsequent collisions start showers of secondary particles which are called Extensive Air Showers (EAS). The first interaction typically takes place at the altitude between 10 and $30 \mathrm{~km}$, depending on the type of primary particle, and continues until the energy of secondary particles are insufficient to continue the process.

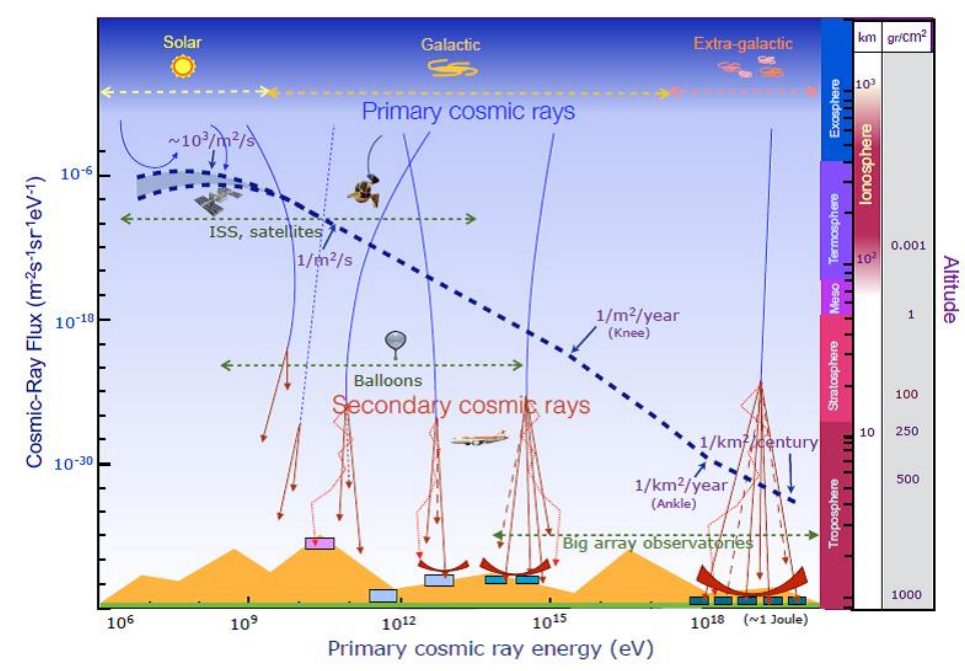

Figure 1: Energy spectrum of primary cosmic rays together with their main detection techniques and sources.

Three major cosmic ray components are distinguished in EAS: electromagnetic, muonic and hadronic. A shower consists in a high energy hadronic core which remain very close to the axis of the shower or the velocity vector of the primary cosmic ray. The hadronic component continually feeds the electromagnetic component producing mesons (mainly pions), which decay in muons, electrons and positrons [1].

Cosmic Ray have several detection techniques according to energies, type of particles, etc. Neutron monitors cover the low energy region and they provide only the integrated flux. Therefore, a large array of this detectors is needed to get an estimation of the arrival direction of the primary cosmic ray. Another type of detectors are based on Cherenkov radiation, which detect 
charged particles but they don't have the capability to distinguis the arrival direction. Muon telescopes cover a higher energy region of the spectrum with the advantage of being able to perform directional studies of the incoming particles. In spite of the fact, that they are very sensitive to space weather events, like magnetic storms, they are quite affected by the temperature profile of the Earth atmosphere on top of the detector, which can't be measured on real time.

As a complement to the techniques described above, TRASGO family detectors offer multitracking capability and, as a consequence, the possibility to detect both individuals and showers of secondary particles [2] [3]. Nowadays, three detectors are already operational and two more are under construction. In this document, a detector of the Trasgo family, TRISTAN, is presented [5]. The main goal is to study different local effects on the secondary cosmic ray flux measured at Earth's surface. Mostly, effects related to the Earth's atmosphere and magnetic field and also space weather events. For that purpose, TRISTAN was installed in Sarmiento de Gamboa's vessel in an temperature controlled room. A two-way journey scan of the cosmic ray flux as a function of the geomagnetic Latitude was performed, crossing the region where the South Atlantic Magnetic Anomaly (SAMA) is located. SAMA is a weakness in Earth's magnetic field over South America and the South Atlantic which allows the inner Van Allen radiation belt to dip down closer to the atmosphere. As a result, much of South America and part of the South Atlantic Ocean get an extra dose of radiation. TRISTAN will be installed at the Livignston Island, close to the Antarctic Peninsula as a part of the Antarctic Comsic Ray Observatory (ORCA).

\section{Antarctic Cosmic Ray Observatory: ORCA}

ORCA consists in a set of detectors with different properties, to measure secondary cosmic rays at the Earth's surface. Two blocks make up the whole observatory. On the one hand, TRISTAN which is based in Resistive Plate Chambers (RPC) technology and built by LIP Coimbra and Hydronav. This detector operated in a temperature controlled room inside the oceanographic ship Sarmiento de Gamboa. On the other hand, there is a neutron monitor (NEMO) together with a muon telescope (MITO) and a mini neutron monitor placed in a container thermally controlled and isolated over Sarmiento de Gamboa's deck. Multiple ship's instruments provide measurements of outdoor temperature, pressure, position, among many other variables.

ORCA has been operating during 48 days along a latitudinal survey from Vigo to Punta Arenas on late 2018, around 100 degrees. NEMO and MITO have been mounted at the Spanish Antarctic Base whereas TRISTAN have done a two-way latitudinal survey on it's way back to Vigo.

\section{TRISTAN Detector}

TRISTAN has been built based on RPC technology. The detector has three active RPC planes built by LIP Coimbra and Hydronav [8]. Each plane has an active size of $1.2 \times 1.5 \mathrm{~m}^{2}$ and the read out is performed by $30 \mathrm{Cu}$ pads instrumented with the fast HADES FEE electronics [4] [7]. Charged particles that travel throughout the upper and second planes will encounter a $1 \mathrm{~cm}$ wide lead layer covering the central $12 \mathrm{Cu}$ pads (around $40 \%$ of the plane surface). The addition of this is to enforce electromagnetic showers associated to gammas and electrons with enough energy to cross the first two planes of the structure. The three planes have been placed at different heights: 
$12.5 \mathrm{~cm}, 39.6 \mathrm{~cm}$ and $60.9 \mathrm{~cm}$. The main performances of the detector for a straight track left by a charged particle are: Time resolution of 300ps and tracking capability of high energy charged particles of.

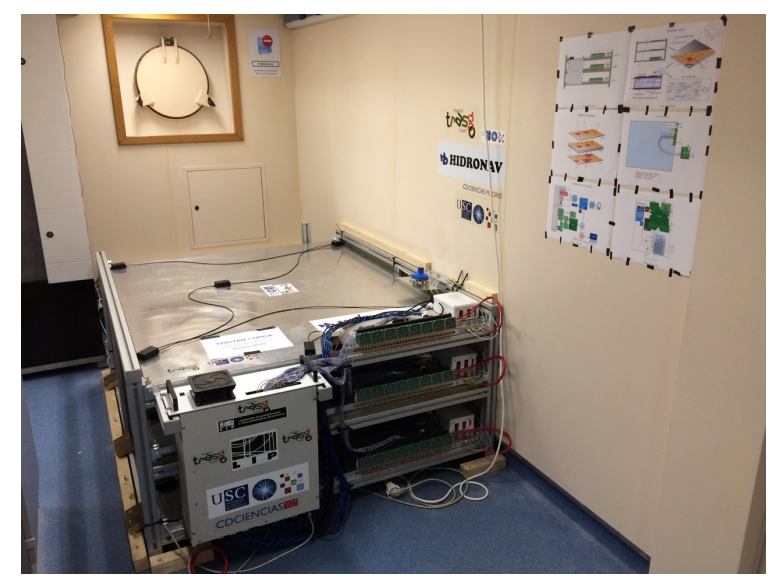

Figure 2: TRISTAN layout on board of the ship Sarmiento de Gamboa during the latitudinal survey on late 2018 and early 2019.

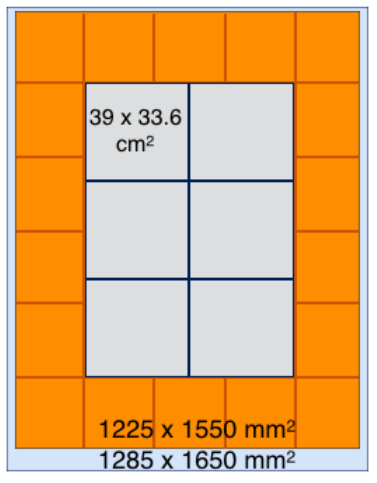

(a)

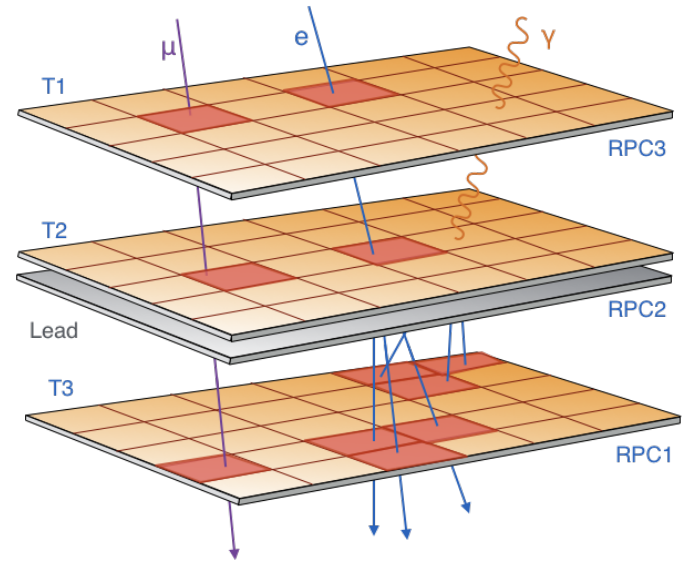

(b)

Figure 3: TRISTAN scheme (a) lead layer between second and third plane from above (b) particle's signals across the active size

The internal layout of each RPC plane is shown in figure 4. Each plane is made by three 2 mm thick layers of glass with a $1 \mathrm{~mm}$ gap in between, placed inside a gas tight methacrylate box. Freon type R134a flows to compensate the small loses of gas. A conductive coating where a high voltage of $\pm 5600 \mathrm{~V}$ is applied covers the glass plates. The size of each pad is $24 \times 25 \mathrm{~mm}^{2}$ and they are separated by straight guard electrodes of $6 \mathrm{~mm}$ thickness to minimize the crosstalk which is almost negligible. 

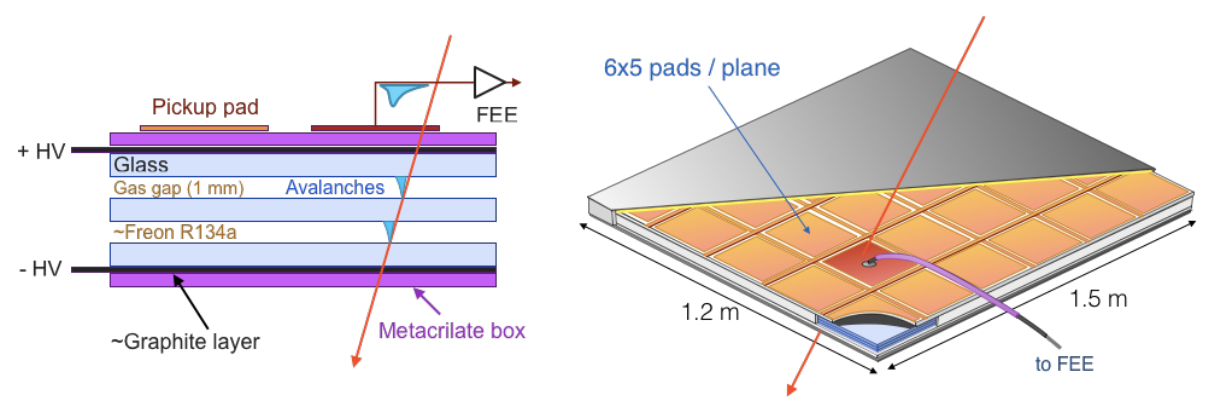

Figure 4: RPC internal layout.

TRISTAN has been designed to use the first 30 channels of the 8 daughterBoards (DBO), which have been connected to one motherboard (MBO) per plane. The data acquisition system (DAQ) is based on the TRBV3 readout board developed at GSI. All MBOs have been connected to the same TRB. The acquisition trigger is done by coincidence between at least one hit in the first and one in the third plane. The detector is managed by an ODROID (single-board computer) which is connected to a Real Time Clock and a Watchdog. The function of the watchdog is to restart the system in case of a connection failed by the ODROID.

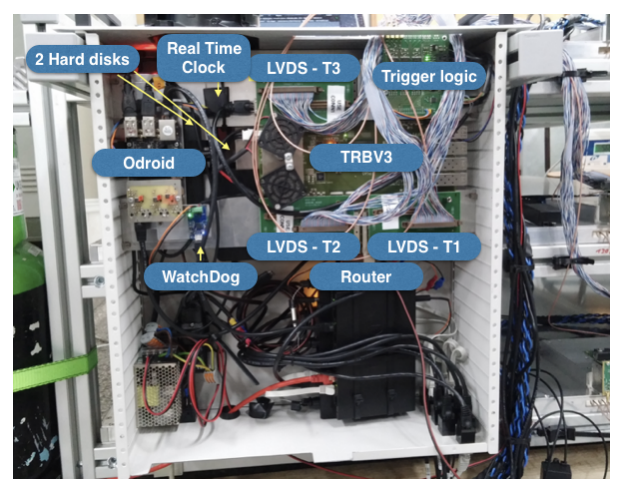

Figure 5: DAQ components and electronic devices.

In addition, a set of sensors have been placed over each plane, five of them are temperature sensors while the other two are for pressure and humidity, respectively. Regarding the high voltage (HV), a HV power supply has been connected to each plane. In the current version, all of them are autocalibrated by temperature and pressure. So that, a small change in these variables is compensated by an increase or decrease of the HV. Finally, the gas flow is permanently monitored by two independent sensors, an optic and a pressure sensor. The optic sensor measure the number of bubbles produced per minute inside the bubblers, whereas the other one measures changes in pressure.

The TRISTAN collaboration main goal is to understand better the secondary cosmic ray showers and to go deeper in the relationship between cosmic rays in a broad range of energies and different phenomena related with the Earth's environment. 


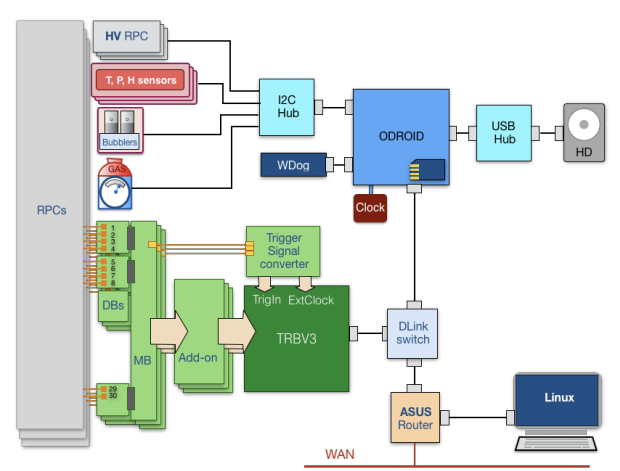

(a)

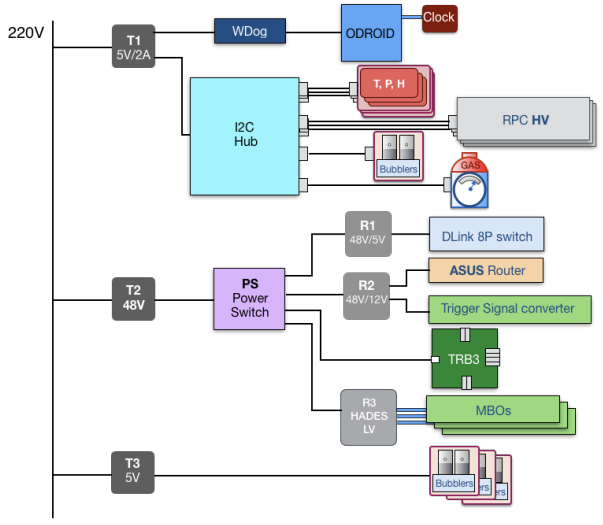

(b)

Figure 6: TRISTAN diagrams of(a) power distribution (b) data and logic flow

\section{TRISTAN: First Results}

TRISTAN has been taking data in a two-way journey on board of the oceanographic ship Sarmiento de Gamboa from Vigo (Spain) to Punta Arenas (Chile) before being installed in the Spanish Antarctic Base on late 2019. It was placed under the ship's deck and surrounded by a thick metallic layer due to the vessel structure. The detector was located in a temperature controlled room.

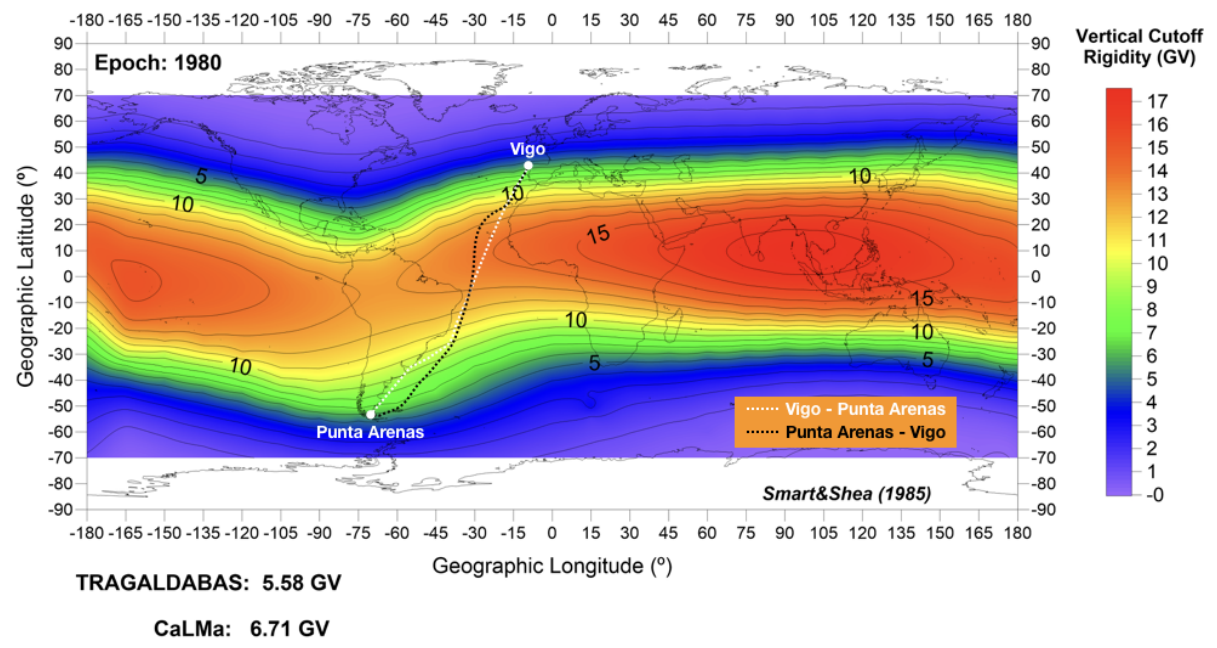

Figure 7: Two-way journey over the Vertical Cutoff Rigidity (GV) map.

During the first trip to Punta Arenas, from November 17th to December 10th a failure in the air conditioning system have been noticed. So that, cosmic rays rate has been measured in different conditions and the corrections needed are very important. In figure 8, the efficiency during the journey is shown and the temperature effect is really clear and that explains the drop in the efficiency around 27th of November. An increase in the temperature inside the room where TRISTAN was located implied an increase in the currents inside the RPC planes, that derived in a higher number of random coincidences detected. The detector has been taking data with 
coincidence trigger between the upper and lower planes, at a rate of around 10 million of events per day $(120 \mathrm{~Hz})$.
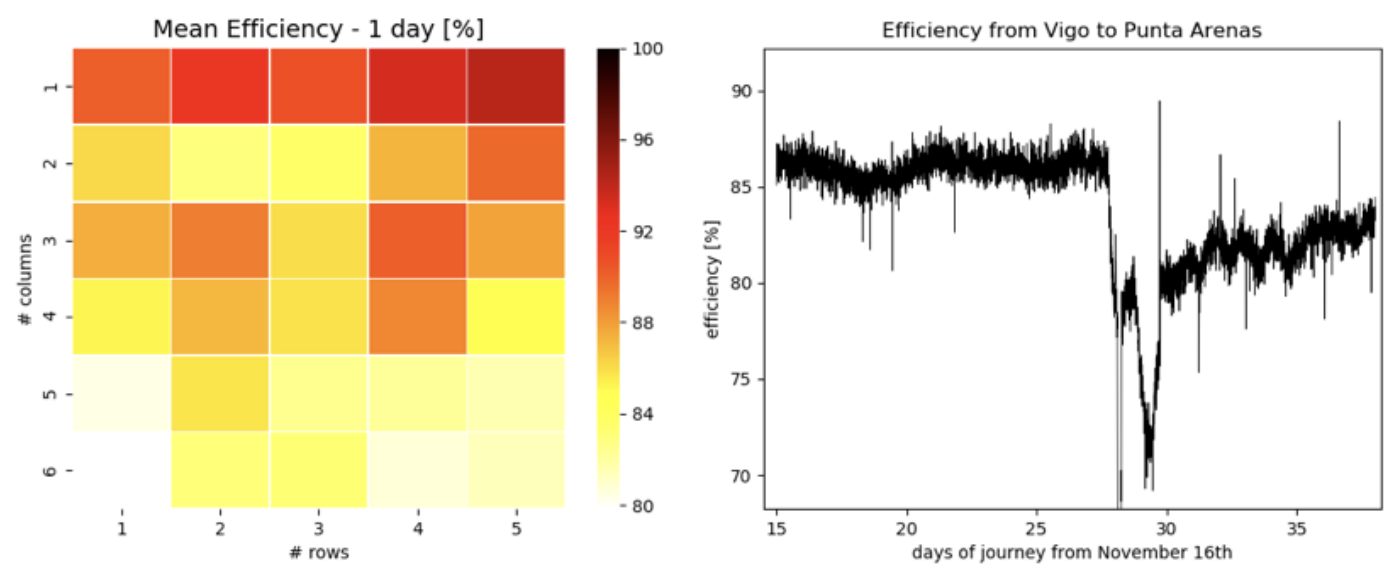

Figure 8: First scan from Vigo to Punta Arenas. Left) mean efficiency along 1 stable day cell by cell. Right) efficiency before and after the air conditioning failure during the journey.

The data taken along both journeys is of special interest because of the wide range of rigidity it covers and the different atmospheric effects to which the detector was exposed.
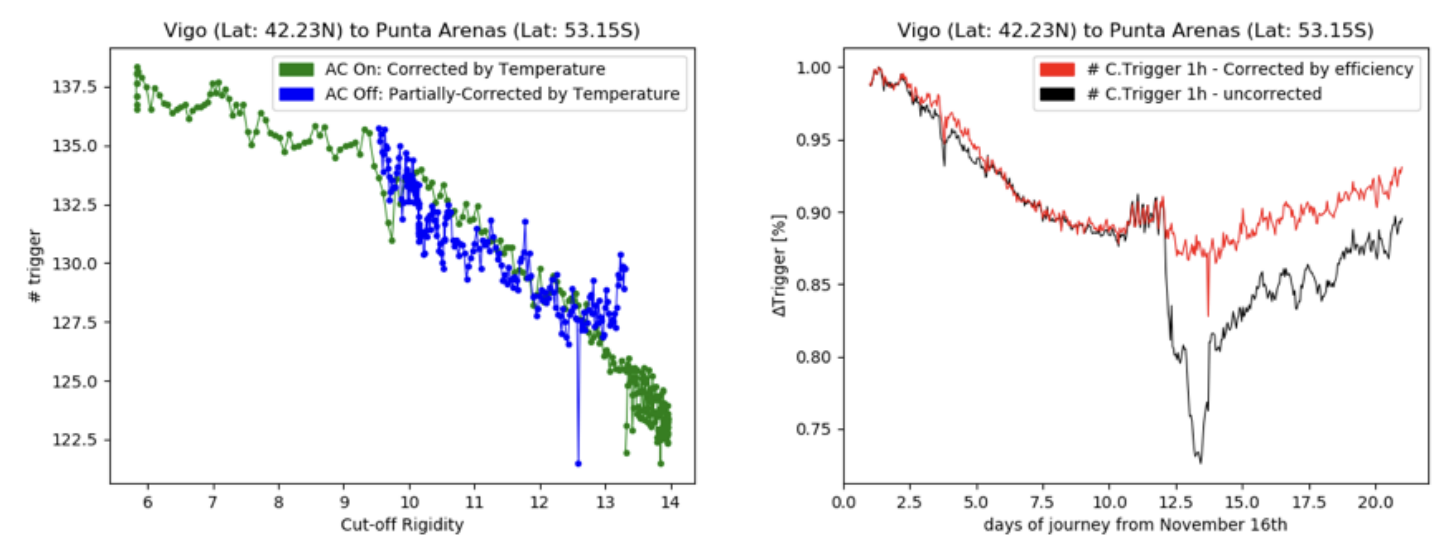

Figure 9: First scan from Vigo to Punta Arenas. Left) Comparison between uncorreted and corrected data by the efficiency effect. Right) Cosmic Rays rate as a function of the rigidity.

The second latitudinal scan, which have been done on early 2019 from Punta Arenas to Vigo is still under study. The detector is now operative in facilities of the University of Santiago de Compostela, Spain, and will be sent to the Antarctica at the end of this year, in order to be installed in ORCA as a permanent detector. 


\section{Conclusions}

In order to carry out a concise and detailed analysis of the correlations between the cosmic ray flux measured at the ground level with the temperature of the Earth's atmosphere at different pressure levels, the Earth's magnetic field and several space weather effects, a detector of the TRASGO family, called TRISTAN has been developed and commissioned. TRISTAN has been designed based on RPC technology and offers multitracking capabilities together with a time resolution around 300ps. The detector has performed a latitudinal two-way scan on board of the oceanographic ship Sarmiento de Gamboa on late 2018 and early 2019 from Vigo to Punta Arenas, respectively. The first results of TRISTAN have been discused in the main document, showing the different conditions in which the detector have been operating. As a consequence, the efficiency of the detector plays an important role and will be studied even deeper in order to study in detail the correlations between a wide range of variables as temperature of the Earth's atmosphere, magnetic field and space weather effects like magnetic storms. The detector will be sent to the Antarctica at the end of this year, in order to be installed in ORCA as a permanent detector.

\section{Acknowledgments}

Authors thanks all the members of the TRISTAN project for their high engagement and also acknowledge the financial support provided by the C. Desarrollo de las Ciencias, of Madrid to the Trasgo project. The TRISTAN project is being funded by the Spanish Agencia Estatal de Investigación under the contract CTM2016-77325-C2-2-P and by the European Regional Development Fund, FEDER. D. García-Castro thanks to the Ministerio de Ciencia, Investigación y Universidades and the European Social Fund (FSE) for a predoctoral grant (FPI 2017).

\section{References}

[1] Gaisser, T.K. (1990). Cosmic rays and particle physics. Cambirdge University Press.

[2] D. Belver et al., TRASGO: A proposal for a timing RPCs based detector for analyzing cosmic ray air showers, Nucl.Inst.Meth. A, 661 (2012) S163-S167

[3] H. Álvarez-Pol et al., Tragaldabas: A new high resolution detector for the regular study of cosmic rays, J.Phys.:Conf.Ser. 632 (2015), no. 1, 012010

[4] Korkanov, G. (2012). New advances and developments on the RPC-TOF wall of the HADES experiment at GSI. PhD thesis. University of Santiago de Compostela.

[5] Garzón, J.A. et al. (2014). TRAGALDABAS: a new RPC based detector for the regular study of cosmic rays. IOP Publishing Ltd and Sissa Medialab srl doi:10.1088/1748-0221/9/09/C09027.

[6] Dimitrieva, A.N. et al. (2010). Corrections for temperature effect for ground-based muon hodoscopes. Astroparticle Physics, Volume 34, Issue 6, p. 401-411. Elsevier B.V. doi:10.1016/j.astropartphys.2010.10.013

[7] I. Frohlich et al., A General Purpose Trigger and Readout Board for HADES and FAIR-Experiments, IEEE Trans. Nucl. Sci. 55 (2008) 59.

[8] L. Lópes et al., Study of RPCs for autonomous field stations in cosmic ray research, PoS(RPC2012)043. 